\title{
Utility of ovarian biopsy in pancreatic metastasis of high-grade serous ovarian carcinoma: A case report
}

\author{
KOHEI NAKAMURA ${ }^{1}$, KENTARO NAKAYAMA $^{1}$, MASAKO ISHIKAWA ${ }^{1}$, \\ NORIYOSHI ISHIKAWA ${ }^{2}$, MAMIKO NAGASE ${ }^{2}$, HIROSHI KATAGIRI ${ }^{1}$, TOMOKA ISHIBASHI ${ }^{1}$, \\ EMI SATO $^{1}$, KOHJI IIDA ${ }^{1}$, RAZIA SULTANA ${ }^{1}$ and SATORU KYO ${ }^{1}$ \\ Departments of ${ }^{1}$ Obstetrics and Gynecology and ${ }^{2}$ Organ Pathology, \\ Shimane University Faculty of Medicine, Izumo, Shimane 6938501, Japan
}

Received September 10, 2015; Accepted February 3, 2016

DOI: $10.3892 /$ mco.2016.886

\begin{abstract}
It is very rare that ovarian carcinoma metastasizes to the pancreas, and pathological diagnosis is required to confirm the primary site. The present study reported a 73-year-old woman with serous carcinoma of the ovary that metastasized to the tail of the pancreas. Metastasis was confirmed by pathological and immunohistochemical examination of a biopsy of the ovarian tumor, an endoscopic ultrasound-guided fine-needle aspiration biopsy of the pancreatic tumor and computerized tomography-guided paraaortic lymph node biopsy. A biopsy of the ovarian tumor is useful to make a precise diagnosis and to determine proper treatment when ovarian and pancreatic tumors are identified at the same time and the primary neoplasm is uncertain.
\end{abstract}

\section{Introduction}

The majority of malignant pancreatic tumors are primary pancreatic neoplasms. Pancreatic metastases are very uncommon $(1,2)$ and usually originate from the kidney, lung, breast, gastrointestinal tract, or from melanoma. Metastasis from the ovary is extremely rare. Most patients with metastatic pancreatic tumors have widespread disease and are, therefore, not candidates for resection. A previous study determined that, although patients with metastatic pancreatic tumors originating from renal-cell cancer can survive for a long time following metastasectomy, survival is less favorable for other primary tumors, including ovarian cancer (3). Only a few cases of ovarian cancer metastasizing to the pancreas have been reported in the literature $(4,5)$.

Differences exist in the treatment of patients with primary ovarian cancer and those with primary pancreatic cancer.

Correspondence to: Dr Kentaro Nakayama, Department of Obstetrics and Gynecology, Shimane University School of Medicine, 89-1 Enyacho, Izumo, Shimane 6938501, Japan E-mail:kn88@med.shimane-u.ac.jp

Key words: ovarian carcinoma, metastatic pancreatic tumor, biopsy
Therefore, it is important to confirm the precise diagnosis when ovarian and pancreatic tumors are discovered simultaneously. The present study reported a patient with serous carcinoma of the ovary that metastasized to the tail of the pancreas. This diagnosis was confirmed by needle-aspiration biopsy of the ovarian tumor, endoscopic ultrasound (EUS) fine-needle aspiration (FNA) biopsy of the pancreatic tumor and computed tomography (CT)-guided paraaortic lymph-node biopsy.

\section{Case report}

A 73 -year-old, gravidity 2 parity 2 , presented to an outside institution complaining of abdominal distension. The patient had a history of interstitial pneumonia and was taking prednisolone. No other significant past medical or surgical history was known. Written informed consent was obtained from the patient and a general physical examination revealed normal results. The hemoglobin level was $6.6 \mathrm{~g} / \mathrm{dl}$ and her cancer antigen (CA)125 level was highly elevated at $382 \mathrm{ng} / \mathrm{ml}$. The carbohydrate antigen 19-9 and carcinoembryonic antigen (CEA) levels were slightly elevated at $52 \mathrm{U} / \mathrm{ml}$ and $15.7 \mathrm{ng} / \mathrm{ml}$, respectively.

Contrast-enhanced CT revealed paraaortic lymphadenopathy, a left ovarian mass and a $6 \mathrm{~cm}$ pancreatic mass (Fig. 1). The patient underwent a CT-guided paraaortic lymph-node biopsy. This revealed a background of sclerotic and edematous stoma, the tumor cells proliferated with papillary and glandular structures, and exhibited hyperchromatic nuclei, heterogeneity in nuclear shape and size with conspicuous nucleoli (Fig. 2A). Taken together, these findings were consistent with a diagnosis of grade 2 serous carcinoma. The cells were positive for cytokeratin (CK)7 (Fig. 2B), CA125 (Fig. 2C), estrogen receptors (ER), BerEP4 and Wilms' tumor protein (WT)-1. They were negative for CK20, CK5/6, caudal type homeobox (CDX)2, thyroid transcription factor-1, carbohydrate antigen 19-9, progesterone receptors (PR), p53, calretinin, and gross cystic disease fluid protein 15 . These immunohistochemical findings were consistent with an ovarian tumor origin.

The patient was referred to Shimane University Faculty of Medicine for further examination and treatment. Magnetic resonance imaging (MRI) and a metastatic workup, including positron emission tomography (PET) were performed. PET-CT revealed abnormal fluorodeoxyglucose uptake in the area of the 

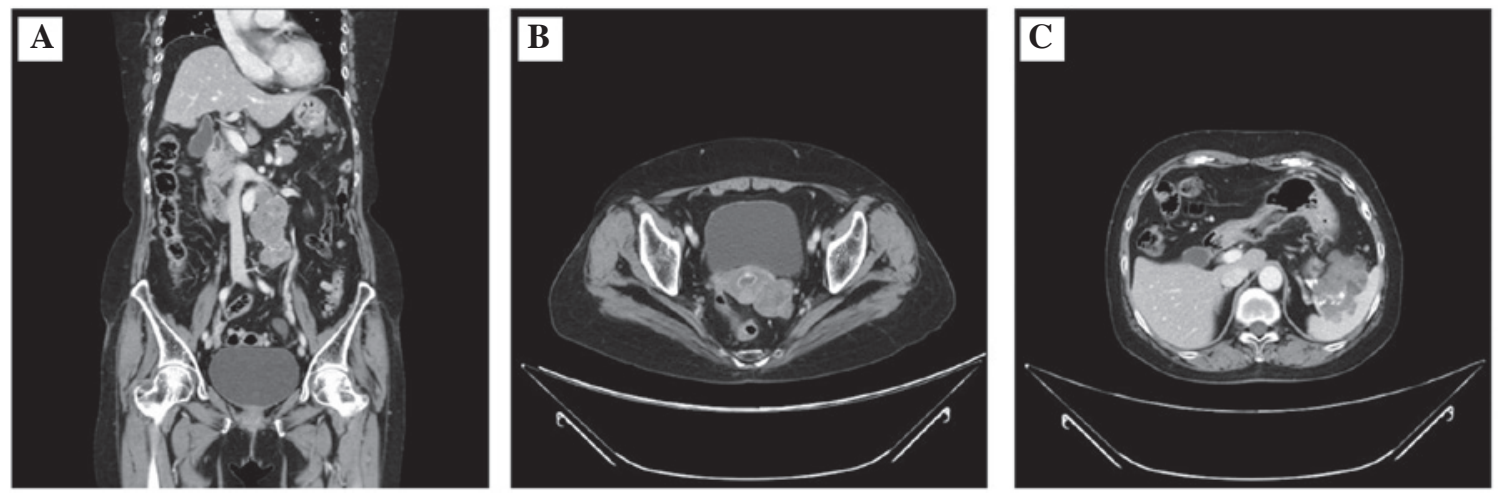

Figure 1. Contrast-enhanced computed tomography of the pelvis. Images of (A) paraaortic lymphadenopathy, (B) left ovarian tumor and (C) pancreatic tumor.

B

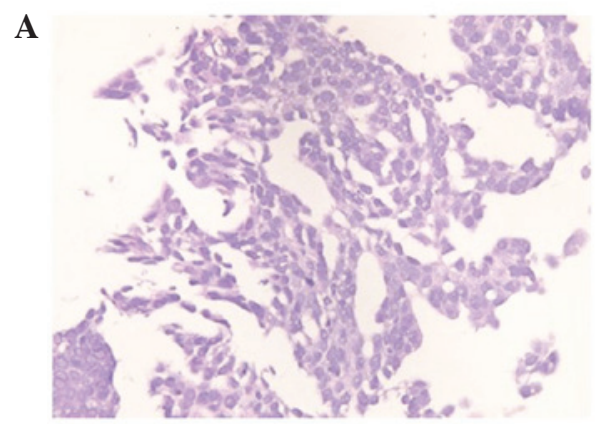

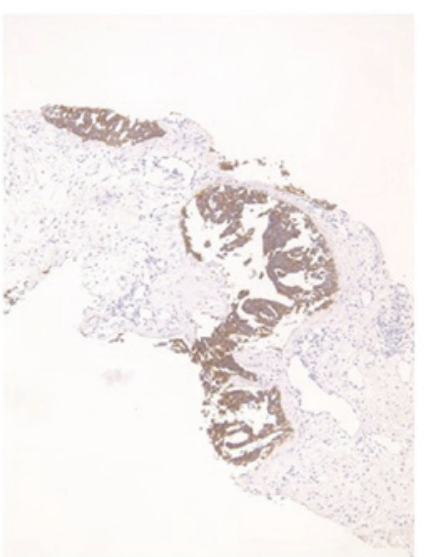

C

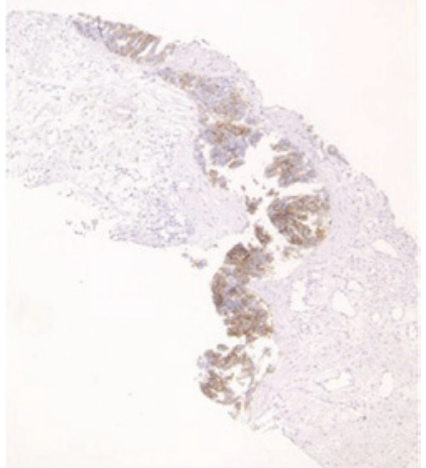

Figure 2. Paraaortic lymph-node smear. (A) The tumor displayed proliferation with papillary and glandular structures, background of sclerotic and edematous stoma and exhibited hyperchromatic nuclei, heterogeneity in nuclear shape and size with conspicuous nucleoli. Positive immunohistochemical staining for (B) cytokeratin 7 and (C) cancer antigen 125.

paraaortic lymph nodes, the ovarian tumor and the pancreatic tumor. MRI revealed a $6 \mathrm{~cm}$ complex cystic mass in the area of the tail of the pancreas. The possible diagnoses included primary ovarian carcinoma with a metastatic pancreatic tumor, primary pancreatic cancer with a metastatic ovarian tumor, and double primary cancers of the ovary and pancreas.

EUS-guided FNA cytology was performed in order to further evaluate the pathology of the pancreatic mass. This revealed a background of coagulation necrosis, that the tumor cells proliferated with papillary to micropapillary and small tubular structures. Psammoma bodies were also observed. Nuclei of tumor cells are hyperchromatic, with an irregular shape and size, with conspicuous nucleoli (Fig. 3A). The tumor cells stained positive for CK7 (Fig.3B), CK20, CA125 (Fig. 3C) and ER. PR and WT-1 were weakly positive, and carbohydrate antigen 19-9 and CD10 were partially expressed. The cells were negative for maspin, CDX2 and CEA. Notably, these findings were consistent with an ovarian origin. Together with the paraaortic findings, the likely diagnosis appeared to be grade 2 serous carcinoma from an ovarian origin.

To confirm the origin of these malignancies, a needle biopsy of the ovarian tumor was performed. The smear revealed serous carcinoma (Fig. 4A) and the results of immunostaining were similar to those of the paraaortic lymph node. The tumor cells were stained positive for CK7 (Fig. 4B), and weakly positive for CK20, CA125 (Fig. 4C), CA19-9, WT-1, and CD10. The cells were negative for p53, maspin, CDX2 and CEA. These pathological and immunohistochemical findings proved to be a match for the ovarian tumor. It was concluded that the mass most likely originated from the ovary and not the pancreas.

Curative surgical therapy was contraindicated in this patient as a result of the location of the metastasis, the tail of the pancreas, an area very difficult to surgically resect. The patient was treated with taxol and carboplatin chemotherapy. At the time of this report, the patient is alive and tolerating her chemotherapy well.

\section{Discussion}

Ovarian cancer is the most commonly fatal gynecological malignancy in Europe and the United States (6). These tumors can metastasize by the intraperitoneal route, the hematogenous route and through lymphatic channels (7), with common metastasis sites, including the pleura, liver, skin, lungs, central nervous system, spleen, bone and breast. It is very rare for ovarian cancer, or for any cancer, to metastasize to the pancreas $(1,2)$. When pancreatic metastases do occur, they usually originate from the kidney, lung, breast, gastrointestinal tract, or from melanoma.

It is highly important to confirm whether a pancreatic tumor is primary or secondary, since treatment differs according to the origin of the malignancy. However, given the low incidence of pancreatic metastasis, it is very difficult to differentiate a 

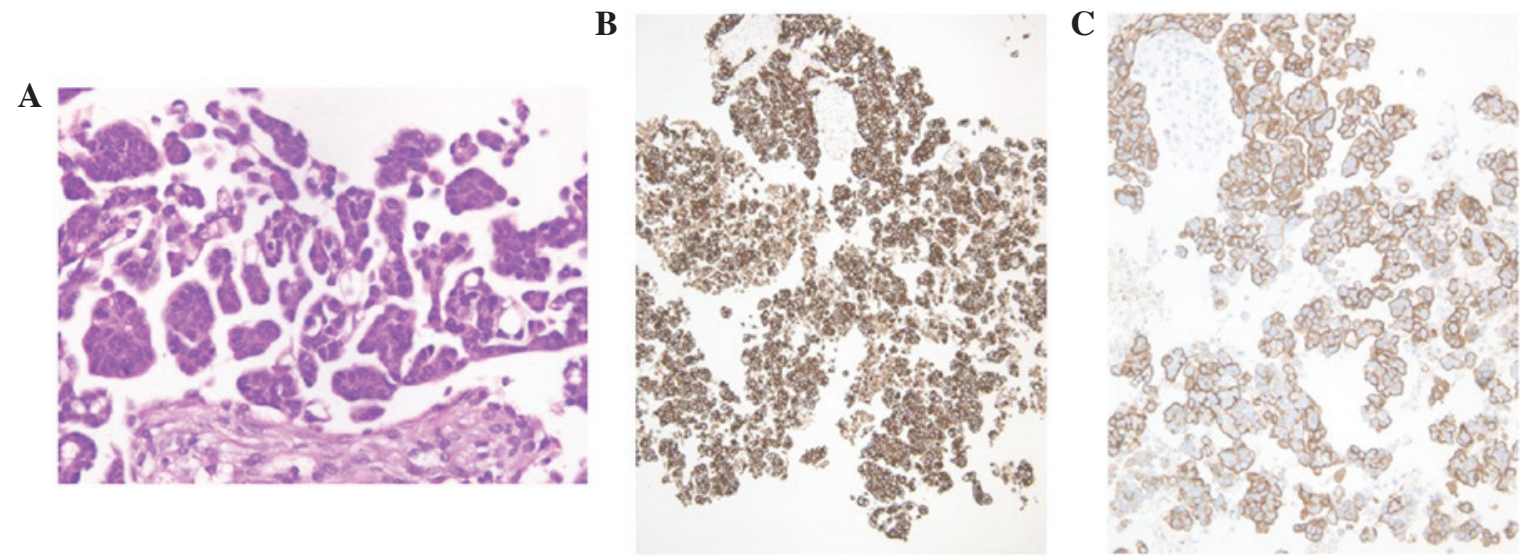

Figure 3. Pancreatic tumor smear. (A) The tumor exhibited proliferation with papillary to micropapillary, and small tubular structures, background of coagulation necrosis and exhibited psammoma bodies. Positive immunohistochemical staining for (B) cytokeratin 7 and (C) cancer antigen 125.

A

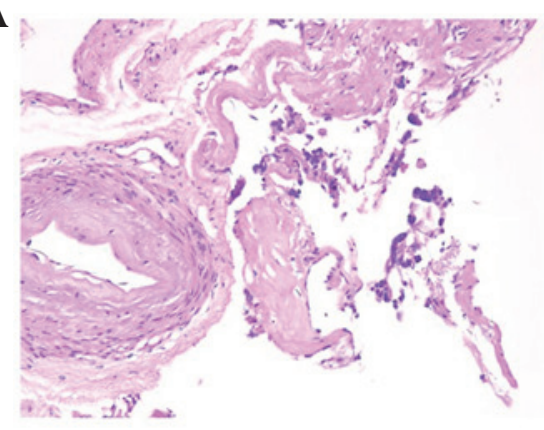

B

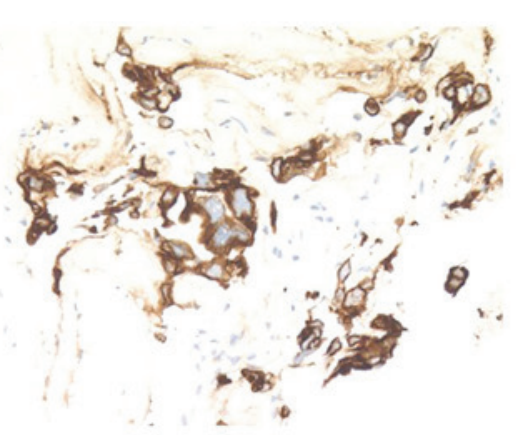

C

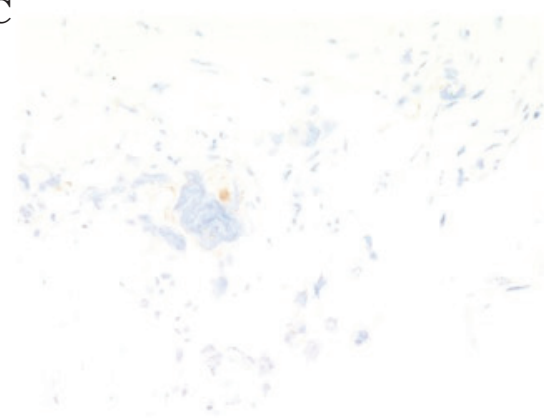

Figure 4. Ovarian tumor. (A) Serous carcinoma. Positive immunohistochemical staining for (B) cytokeratin 7 and weak positive immunohistochemical staining for $(\mathrm{C})$ cancer antigen 125.

primary pancreatic neoplasm from a metastatic malignancy. The symptoms and signs are similar, and radiological imaging is unable to differentiate primary from secondary lesions (8).

In the present patient, pathological and immunohistochemical examination of a needle-aspiration biopsy of the ovarian tumor, CT-guided paraaortic lymph biopsy and EUS-guided FNA biopsy of the pancreatic tumor were helpful to confirm the diagnosis. A biopsy of a metastatic tumor can reveal a highly likely place of origin; however, it is not precise. To confirm the origin of a tumor, biopsy of the likely culprit is required. Unfortunately, performing an ovarian-tumor biopsy is generally contraindicated owing to the risk of disseminating malignant cells. In the present patient, an ovarian origin would mean that the carcinoma was International Federation of Gynecology and Obstetrics stage IV. Therefore, dissemination would not change the staging, and the present study was comfortable performing a biopsy of the ovarian tumor to obtain a definitive diagnosis.

It is rare that pancreatic resection is indicated for a metastatic tumor (3). The procedure has not been shown to improve survival or quality of life (3). In the present patient with ovarian cancer and a metastatic pancreatic tumor, chemotherapy was selected as the primary treatment as a result of the great difficulty in surgically resecting the metastatic location, the tail of the pancreas.

Pathological and immunohistochemical examination of a lymph-node biopsy, an EUS-guided FNA biopsy of the pancreatic tumor and needle-aspiration biopsy of the suspected primary tumor is useful to make an exact diagnosis and determine the proper treatment in patients with simultaneous pancreatic and other-site tumors. This is particularly true when it cannot be determined whether the pancreatic tumor is primary or secondary in origin.

\section{References}

1. Hiotis SP, Klimstra DS, Conlon KC and Brennan MF: Results after pancreatic resection for metastatic lesions. Ann Surg Oncol 9: 675-679, 2002.

2. Nakamura E, Shimizu M, Itoh T and Manabe T: Secondary tumors of the pancreas: Clinicopathological study of 103 autopsy cases of Japanese patients. Pathol Int 51: 686-690, 2001.

3. Konstantinidis IT, Dursun A, Zheng H, Wargo JA, Thayer SP, Fernandez-del Castillo C, Warshaw AL and Ferrone CR: Metastatic tumors in the pancreas in the modern era. J Am Coll Surg 211: 749-753, 2010.

4. Gunay Y, Demiralay E and Demirag A: Pancreatic metastasis of high-grade papillary serous ovarian carcinoma mimicking primary pancreas cancer: A case report. Case Rep Med 2012: 943280, 2012.

5. Hadzri MH and Rosemi S: Pancreatic metastases from ovarian carcinoma-diagnosis by endoscopic ultrasound-guided fine needle aspiration. Med J Malaysia 67: 210-211, 2012.

6. Wingo PA, Tong T and Bolden S: Cancer statistics, 1995. CA Cancer J Clin 45: 8-30, 1995.

7. Rose PG, Piver MS, Tsukada Y and Lau TS: Metastatic patterns in histologic variants of ovarian cancer. An autopsy study. Cancer 64: 1508-1513, 1989.

8. Charnsangavej $\mathrm{C}$ and Whitley NO: Metastases to the pancreas and peripancreatic lymph nodes from carcinoma of the right side of colon: CT findings in 12 patients. AJR Am J Roentgenol 160: 49-52, 1993. 